\title{
A CRISPR definition of genetic modification
}

\author{
Gene editing techniques have the potential to substantially accelerate plant breeding. Now, officials in the United \\ States and Europe are arguing that it is not genetic modification - and that is a good thing!
}

G enome engineering using CRISPRCas9 technology has come a long way in a very short time. It was in the late 1980s that clustered regularly interspaced short palindromic repeats (CRISPRs) were identified in DNA from Escherichia coli, and the early 1990s when CRISPR-associated systems (CASs) were seen to be widespread within prokaryotes, but these systems remained a curiosity for almost 20 years, chiefly studied for their role in bacterial antiviral defence. A little over five years ago, the system was successfully engineered into a highly adaptable, targetable tool for cleaving and rewriting DNA sequences and was named Science magazine's breakthrough of the year in 2015, and given an entry in Merriam-Webster's dictionary last year. Few can even remember the much lauded approaches to genome editing that were so much talked about in the years B.C. (before CRISPR), such as TALEN's and zinc finger nucleases. Now, CRISPR-Cas9 has passed another milestone with the decision by the United States Department of Agriculture (USDA) to clear a crop developed using CRISPR for commercial growth without regulation as a genetically modified organism (GMO).

The crop in question is a mushroom developed by Yinong Yang at Pennsylvania State University. These are common white button mushrooms (Agaricus bisporus), that have had their polyphenol oxidase (PPO) enzymes disabled. Yang achieved this by using CRISPR to remove a small number of base pairs from one of six PPO genes in the mushroom's genome, reducing its PPO activity by around $30 \%$. The ultimate effect of this change is to produce a mushroom that remains white for longer when cut rather than turning brown, greatly extending its shelf life. These mushrooms were developed in 2015, and in 2016 the USDA's Animal and Plant Health Inspection Service (APHIS) gave the opinion that they would not be subject to USDA regulation. The announcement by U.S. Secretary of Agriculture, Sonny Perdue, on the 28 March confirms this opinion and clarifies the situation for other gene-edited crops.

The United States takes an essentially functional view of GMOs, not concerning itself with the technologies that created the animals or plants but rather what the effects of the manipulations have been.
The USDA statement further clarifies,

"Under its biotechnology regulations, USDA does not currently regulate, or have any plans to regulate plants that could otherwise have been developed through traditional breeding techniques as long as they are developed without the use of a plant pest as the donor or vector and they are not themselves plant pests". Under this ruling genetic deletions, single base-pair substitutions and even the introgression of nucleotide sequences from related plants that could potentially have come about through cross breeding, are all outside the scope of USDA regulation. Furthermore, offspring of genetically modified parents will also be exempt, as long as the genetic modification of the parents is not retained by those offspring.

Yang's mushrooms may be the test case for the USDA's approach, but there are plenty more crops under development that have benefited from the intervention of CRISPR and so should be subject to the same exemption. Disease- and droughtresistant corn, canola oil with increased nutritional value, herbicide-resistant soybeans, potatoes more resistant to cold storage, gluten-free wheat, and much more are all possible using CRISPR-Cas9 editing. But, if the USDA does not class these as GMOs, then they (and any processed products containing them) will not need to be labelled as such.

Genetic modification (GM) labelling is a contentious issue, particularly as there is no agreed definition of GM. Equally, while it is generally assumed that labelling will reduce the attractiveness of products to consumers, there are few well-controlled studies on the subject and the scant data fail to identify substantial effects of labelling on consumer behaviour. Supporters of labelling argue that it supports transparency and enhances consumer choice; while those opposed say that it would be cumbersome, bureaucratic, and only serve to increase the price of food and mislead consumers.

In Europe, the labelling of 'GM foods' is legally mandated. The current rules came into force in 2004 , long before genome editing, let alone CRISPR-Cas9, was a viable technique. Unlike in America, where the genetic material of organisms determines their status, in Europe a GMO is any organism that has been created using GM technology. Food has to be labelled as GM if any of the ingredients come from a GMO, even if no GM material is present in the final product, as is the case with flour or oils. Somewhat illogically, however, products produced with GM technology, for example cheese made with GM microorganisms, or the products of animals fed on GM-labelled feed, are not themselves labelled. These regulations apply to imported food as well as produce from within Europe, which could cause considerable difficulties with, say, non-browning mushrooms from America that the USDA would not consider to be GM but Europe would.

However, European regulations do not consider 'mutagenesis' to be a GM technology, so plants and animals carrying novel traits produced by nuclear radiation or chemical mutagens are not GMOs. This exemption has become crucial to the debate over what is and is not a GMO in Europe, with the recent publication of an initial opinion from Michel Bobek, Advocate General of the Court of Justice of the European Union. In this, he reaches the opinion that "an organism obtained by mutagenesis can be a GMO" if it is altered in a way that does not occur naturally by mating and/or natural recombination. However, he also suggests that the mutagenesis exemption should not be confined to 'safe' mutagenesis techniques (radiation and chemical mutagens), as they were understood in 2001 when the original European GMO directive was drafted, but that new techniques that induce mutagenesis should also count. Zinc finger nucleases, TALENs and CRISPR-Cas9 are all mentioned as capable of causing targeted mutagenesis suggesting that at least some of their applications should not be classed as GM.

This will not be the end of the debate. Bobek was asked to address the question in the first place at the behest of nine French, non-governmental organizations opposed to GMOs who presumably hoped to restrict the mutagenesis exemption to pre-2001 technologies. However, with the USDA decision and Bobek's opinion it seems that legislatures on both sides of the Atlantic may be edging towards a more consistent and rational approach to modern genomic engineering.

Published online: 3 May 2018 https://doi.org/10.1038/s41477-018-0158-1 